\title{
Short-term effects of transdermal estradiol in men undergoing androgen deprivation therapy for prostate cancer: a randomized placebo-controlled trial
}

\author{
Nicholas Russell1,2, Rudolf Hoermann', Ada S Cheung ${ }^{1,2}$, Michael Ching ${ }^{3}$, \\ Jeffrey D Zajac 1,2, David J Handelsman ${ }^{4}$ and Mathis Grossmann 1,2 \\ ${ }^{1}$ Department of Medicine (Austin Health), The University of Melbourne, Heidelberg, Australia, ${ }^{2}$ Department of \\ Endocrinology, ${ }^{3}$ Pharmacy Department, Austin Health, Heidelberg, Australia, and ${ }^{4}$ ANZAC Research Institute, \\ University of Sydney, Concord Hospital, Concord, NSW, Australia
}

\author{
Correspondence \\ should be addressed \\ to N Russell \\ Email \\ nicholas.russell@austin.org.au
}

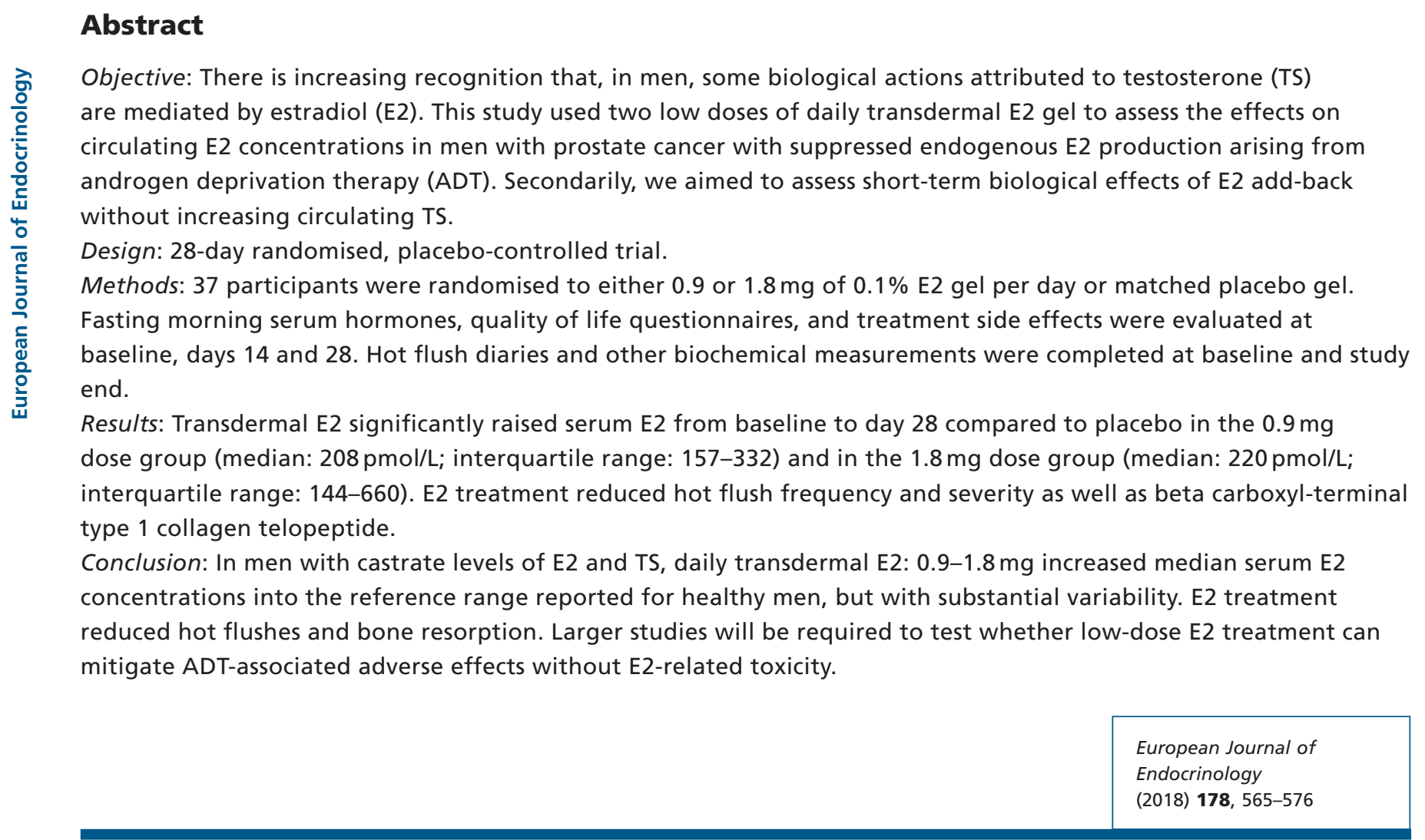

\section{Introduction}

The benefits for disease control and the bone and metabolic adverse effects of androgen deprivation therapy (ADT) with gonadotropin-releasing hormone (GnRH) analogues in the treatment of prostate cancer (PCa) are established $(1,2)$. GnRH analogues produce castrate levels of testosterone (T), and its metabolite estradiol (E2) in men. Over decades since Huggins' Nobel Prize-winning report of castration as an effective palliative treatment for advanced prostate cancer in the 1940s, surgical castration has progressively been replaced by medical castration, 
initially using high-dose oral estrogens but more recently using GnRH analogs to achieve castrate T levels $(3,4)$. High-dose oral estrogen treatment has been largely superseded due to increased thromboembolic events attributed to hepatic first pass metabolism of ingested estrogens with consequent upregulation of liver-derived clotting factors $(3,5)$.

The long natural history of PCa combined with recognition that some components of medical castration are due to estrogen deficiency as well as androgen deficiency (6) has renewed interest in the use of non-oral E2 to mitigate the bone, brain and metabolic adverse effects of $\operatorname{ADT}(7,8)$. Two alternative approaches are the use of high-dose transdermal E2 as a sole mode of ADT (replacing GnRH analogs) or low-dose E2 'add-back' in addition to GnRH analogs to restore E2 to levels sufficient to mitigate adverse effects (9). The former approach is used in the PATCH study, a phase 2 single-blinded randomised controlled trial (RCT) designed to assess the safety and efficacy of ADT comparing high-dose E2 via transdermal patches alone to produce castrate $\mathrm{T}$ levels with standard ADT using GnRH analogs (8). Only two small RCTs, enrolling 12-25 men have tested the low-dose E2 'addback' approach $(10,11,12)$, and these RCTs used oral E2. Transdermal E2, in contrast to oral E2 is not subject to pro-thrombotic hepatic first pass metabolism, however, even transdermal E2, if given at supraphysiologic doses may have other undesirable effects, such as gynecomastia or nausea. Furthermore, little is known about steady-state circulating E2 achieved by transdermal E2 dosing in older men.

This RCT was designed to assess the effects of two low doses of daily transdermal E2 gel (providing respectively 0.9 and $1.8 \mathrm{mg}$ E2 per day) compared with placebo on circulating E2 concentrations in men with $\mathrm{PCa}$ with suppressed endogenous E2 production due to GnRH analog treatment. This provided the opportunity to assess biological effects of E2 occurring in the presence of castrate circulating $\mathrm{T}$ concentrations. We hypothesised that E2 treatment would improve hot flushes and quality of life, reduce biomarkers of bone resorption, reduce insulin resistance and lower insulin-like growth factor 1 (IGF-1).

\section{Subject and methods}

We conducted a 28-day, randomised, blinded, placebocontrolled, multi-arm, parallel-group trial at Austin Health, a tertiary referral hospital affiliated with The
University of Melbourne. Participants were recruited from outpatient clinics from June 2016 to July 2017. Men aged 55-85 years were eligible for the study if they had been receiving GnRH agonists or antagonists for non-metastatic PCa for a minimum of 4 weeks. Exclusion criteria were diabetes mellitus; impaired performance status (Eastern Cooperative Oncology Group Performance Status $(\mathrm{ECOG})>1)$; past or current venous thromboembolism (VTE); breast cancer; human immunodeficiency virus infection; osteoporosis, antiresorptive or strontium ranelate use; systolic blood pressure $>160$ or diastolic blood pressure $>100$; symptomatic heart failure; stroke, transient ischaemic attack, myocardial infarction or angina within 12 months; glucocorticoid treatment; alcohol or illicit drug abuse.

The trial protocol was approved by the local ethics committee (HREC/16/Austin/89) and each participant provided written informed consent. The trial was preregistered with the Australian New Zealand Clinical Trials Registry (identifier 12616000373471). We followed the CONSORT checklist of information to include when reporting a randomised trial.

Participants were randomly allocated, in a concealed fashion, to one of four intervention groups: E2 $1 \mathrm{~mL}$ $(0.9 \mathrm{mg})$ per day or matching placebo $1 \mathrm{~mL}$ per day or E2 $2 \mathrm{~mL}(1.8 \mathrm{mg})$ per day or matching placebo $2 \mathrm{~mL} /$ day. Randomisation (Fig. 1) occurred as follows: first, participants were stratified by body mass index (BMI) into categories below or above the clinic median $(\leq 29$ or $>29 \mathrm{~kg} / \mathrm{m}^{2}$ ); secondly, using simple randomisation (coin toss), participants were then allocated in a 1:1 ratio to a daily gel volume of 1 or $2 \mathrm{~mL}$; thirdly, within each volume group, participants were allocated by restricted randomisation, using blocks of size 3 , to E2 or placebo in a ratio of 2:1. The final allocation to E2 or placebo was performed using a computer-generated randomization scheme administered by an investigator (MC) with no clinical involvement in the trial.

E2 gel was Sandrena $1 \mathrm{mg} / \mathrm{g}$ estradiol (Aspen Pharmacare, St Leonards, NSW, Australia). Placebo gel was a-gel (Fresenius Kabi, North Ryde, NSW, Australia) and matched the E2 gel for colour, smell and consistency. E2 and placebo were re-packaged into identical metereddose syringes by pharmacy and dispensed to patients labelled as 'estradiol or placebo', with instructions to use the specified volume once daily on the skin of the chest or upper arms. This process concealed treatment allocation within each volume group and maintained blinding of participants, investigators and clinicians for the duration of the study. Blinding was removed 


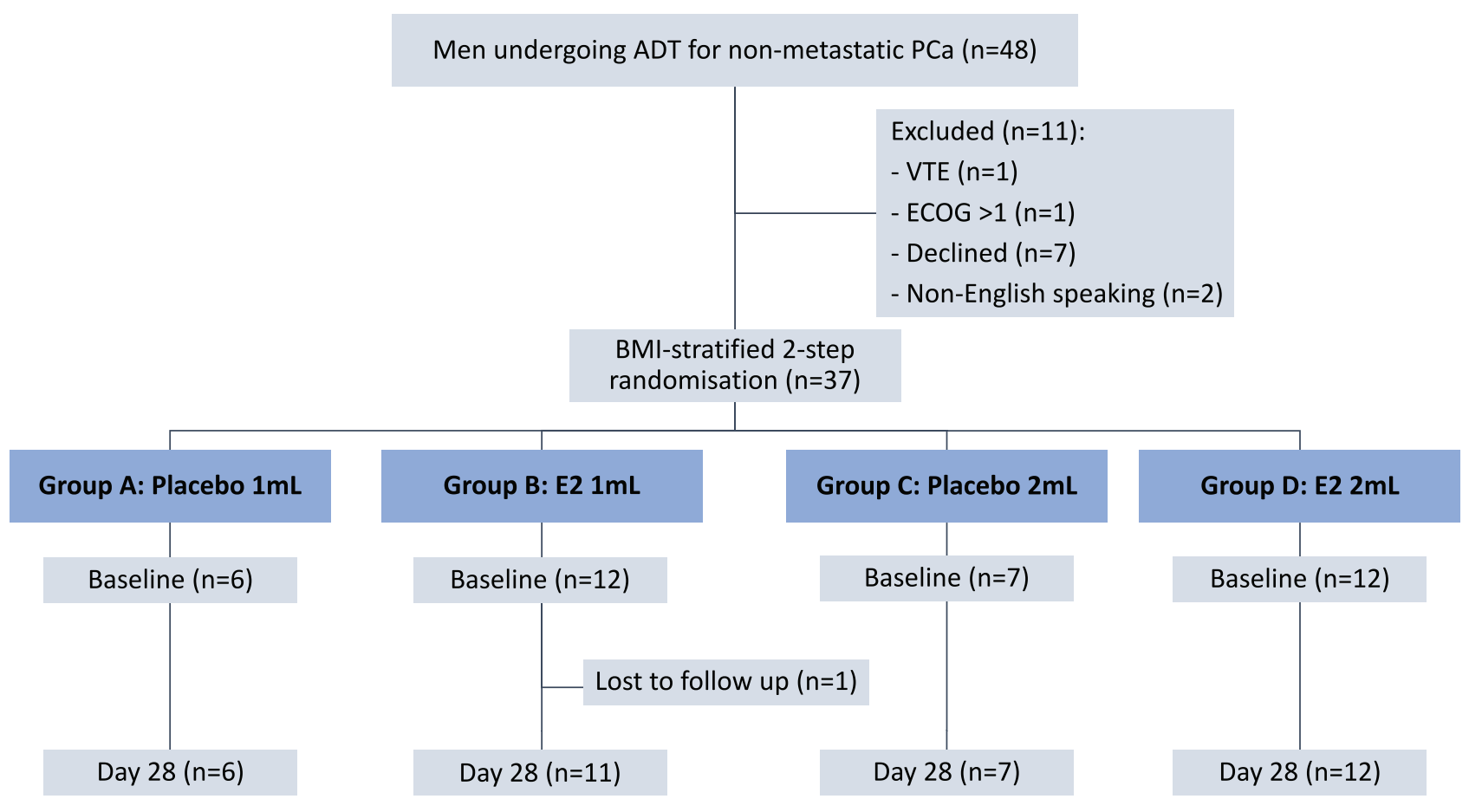

\section{Figure 1}

Study diagram. Participants were stratified by BMI ( $\leq 29$ or $>29$ ). Within each BMI category, men were first randomised by coin toss to gel $1 \mathrm{~mL}$ or $2 \mathrm{~mL}$ (step 1) and secondly, by computer-generated randomisation algorithm, in a 2:1 ratio (block size 3), to E2 $(1 \mathrm{ml} / 2 \mathrm{ml})$ or matching-volume placebo (step 2). Men with BMI above and below the clinic median of 29 were thus equally represented in each of the 4 intervention groups, $A, B, C$, and D.

immediately prior to data analysis, after the database had been cleaned and locked.

Participants were seen at baseline, and days 14 and 28 of the intervention. At each visit, men completed the FACT-P questionnaire (12) and were interviewed to ascertain treatment side effects. FACT-P total score grades quality of life from 0 to 156 , with higher scores indicating better quality of life. Participants completed a hot flush diary as described by Loprinzi et al. (13) for the 7 days prior to gel commencement and during days 21-28 of the intervention. Average daily hot flush score was derived from hot flush frequency and severity as described (13).

Gel syringes were collected at day 28 and residual volume was recorded to assess adherence. Men were given standard written advice recommending a daily dietary calcium intake of $1300 \mathrm{mg}$ and advised not to make changes to their Vitamin D or calcium supplementation status. Adverse events were graded according to Common Terminology Criteria for Adverse Events, version 4.03 (14).

Fasting morning pre-dose blood samples were drawn at each visit. Serum was stored at $-80^{\circ} \mathrm{C}$ for batched analysis of sex steroid profile by liquid chromatography mass spectrometry (LC-MS) (15). Limits of quantification and detection were 18 and $11 \mathrm{pmol} / \mathrm{L}$ respectively for E2; 11 and $4 \mathrm{pmol} / \mathrm{L}$ for estrone (E1); 0.10 and $0.03 \mathrm{nmol} / \mathrm{L}$ for $\mathrm{T}$ and 0.35 and $0.17 \mathrm{nmol} / \mathrm{L}$ for dihydrotestosterone (DHT). CVs were 5-10\%, 4-9\%, 2-8\% and 7-13\% respectively for within-run reproducibility at three levels of quality control samples.

At baseline and day 28, hematology and biochemistry including hemoglobin, hematocrit, luteinising hormone (LH), follicle-stimulating hormone (FSH), insulin, glucose, c-peptide, IGF-1, triglycerides and total and high-density lipoprotein (HDL) cholesterol were measured using standard methodologies at the Biochemistry Department, Austin Health. Updated homeostatic model assessment of insulin resistance (HOMA2-IR) (16) and low-density lipoprotein (LDL) cholesterol were calculated using standard methodologies. At baseline, 25-OH Vitamin D3 was measured by chemiluminescent immunoassay (Roche Cobas 8000, Roche Diagnostic International), with inter assay CV 3.9-19.5\%.

Serum beta carboxyl-terminal type 1 collagen telopeptide (CTX) and pro collagen type 1 amino- 
terminal propeptide (P1NP) were measured at baseline and day 28 on a Roche Cobas e602 platform (Roche Diagnostic International) with inter assay CVs of 2.5-3.9\%. CTX and P1NP are biomarkers reflecting different components of coupled bone remodelling by basic multicellular units, with CTX primarily reflecting osteoclastic activity and P1NP reflecting subsequent osteoblastic bone formation.

\section{Study design and pre-specified outcomes}

The pre-specified primary outcomes were the serum E2 concentration from baseline to day 28 measured by LC-MS and the change in the daily hot flush score. We report serum E2 concentrations in the 4 groups from baseline to day 28 in each E2 group with its respective placebo group and combined the groups for the symptomatic outcome. Pre-specified secondary outcomes were changes, baseline to study end, in other serum sex steroids measured by LC-MS (T, DHT, E1), gonadotrophins, HOMA2-IR, IGF-1, bone remodelling markers, hot flushes and prostate cancer-specific quality of life.

\section{Power analyses}

We determined that 8 men per group would provide the trial with a power of $80 \%$ to detect a difference in circulating E2 concentrations between E2 and placebotreated men based on data from the literature $(10,12)$ reporting a substantial increase in E2 levels of $150 \mathrm{pmol} / \mathrm{L}$ with a pooled standard deviation of $100 \mathrm{pmol} / \mathrm{L}$, at an alpha level of 0.05 . For the secondary outcome of hot flushes, we aimed to detect a change in daily frequency of 7 with a standard deviation of 7 , requiring 17 participants (17). This appeared to be achievable by combining the two E2 groups, provided their outcomes were similar, as expected.

\section{Statistical analysis}

Main outcomes were non-normally distributed, and data are reported as median (interquartile range). Undetectable steroid concentrations were assigned the limit of detection value for data analysis. Betweengroup differences were tested using the exact unpaired Wilcoxon-Mann-Whitney test for two groups, KruskalWallis test for more than two groups or Fisher's exact test in case of frequency data. For further analysis, the two E2 groups showing comparable outcomes were combined to increase power and compared with the combined placebo groups. The combined E2 group is subsequently referred to as the E2 group. We used a robust linear mixed model to account for both within-subject variation and outliers and adjusted for baseline values (18). Degrees of freedom were determined by Satterthwaite's method (19). Outcomes refer to the change from start to the end of the trial between the $\mathrm{E} 2$ group and the placebo group and are reported as robust mean adjusted difference (robust MAD) plus 95\% confidence interval. Two-sided $P$ values $<0.05$ denoted statistical significance. Analyses were conducted using the statistical base package R, version 3.4.3 for Mac (18) with the added packages robustlmm 2.1-4 (19) and lmerTest 2.0-36 (20).

\section{Results}

In our clinic database, we identified 48 men meeting the inclusion criteria of the study. Eleven men were excluded on the basis of prior venous thromboembolism $(n=1)$, ECOG $>1(n=1)$, inability to consent in English $(n=2)$ or refusal to participate $(n=7)$. Thirty-seven were randomised with 1 loss to follow-up after the day 14 visit (Fig. 1).

Baseline characteristics by group are shown in Table 1. Participant age range was $60-83$ years with 18 (49\%) receiving ADT for more than 3 months (median: 3 months, range: 1-120 months). Baseline median $\mathrm{T}$ was $0.2 \mathrm{nmol} / \mathrm{L}$ (range of $0.04-0.9 \mathrm{nmol} / \mathrm{L}$ ) and $\mathrm{E} 2$ was $11 \mathrm{pmol} / \mathrm{L}$ (range undetectable to $65 \mathrm{pmol} / \mathrm{L}$ ). Seven men (19\%) were receiving medication from classes used to treat hot flushes, but these medications or their doses were not altered during the study.

Both doses of E2 significantly raised serum E2 from baseline to day 28 compared to placebo (Fig. 2 and Table 2). Day 28 serum E2 concentration ranged from 106 to $870 \mathrm{pmol} / \mathrm{L}$ in the $0.9 \mathrm{mg}$ dose group (median: 208 pmol/L; interquartile range: 157-332), and $96-1814 \mathrm{pmol} / \mathrm{L}$ in the $1.8 \mathrm{mg}$ dose group (median: $220 \mathrm{pmol} / \mathrm{L}$; interquartile range: 144-660). There was no association between day 28 E2 concentration and participant BMI, age or duration of ADT at enrolment (data not shown). Based on inspection of returned syringes from the 36 participants who completed follow-up, 1003 of 1008 prescribed doses were administered. Because there were comparable E2 concentrations in the placebo groups and in the E2 groups respectively, these groups were combined to increase power in the analysis of secondary outcomes. 
Table 1 Baseline characteristics by group. Data are presented as median (IQR).

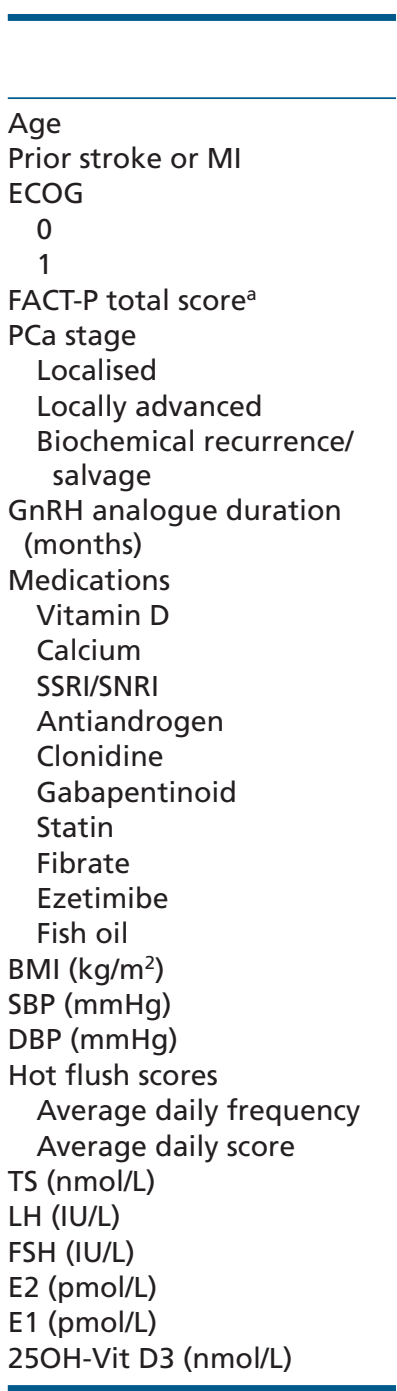

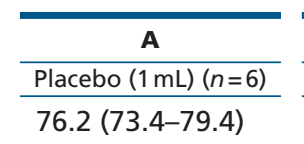

1
6
0
$128(123-133)$

$\frac{\text { B }}{\frac{\mathrm{E} 2(1 \mathrm{~mL})(n=12)}{74.9(68.9-75.5)}}$

$\frac{\text { C }}{\frac{\text { Placebo }(2 \mathrm{~mL})(n=7)}{72.7(66.7-74.5)}}$

2
2
2

$8.5(2.3-18.5)$

2
8
4
$122(118-132)$

3
2
7

$3.0(1.8-10.2)$

3
1
0
0
0
0
2
0
0
1
$29.3(27.6-32.0)$
$138(127-140)$
$80(76-82)$

$2.1(0.00-5.1)$
$2.7(0.00-6.2)$
$0.26(0.22-0.53)$
$0.300 .23-0.82$
$2.9(2.2-4.7)$
$11(11-11)$
$164(134-194)$
$77.0(68.8-106.0)$

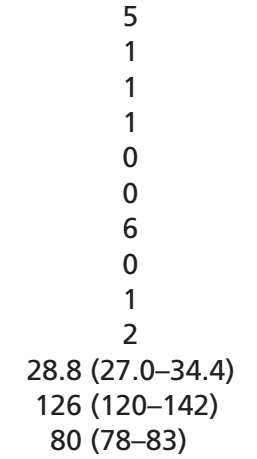

$1.1(0.11-4.1)$

$1.4(0.21-6.6)$

$0.14(0.11-0.22)$

$0.10(0.10-0.32)$

$3.6(2.7-4.3)$

$11(11-11)$

151 (116-216)
77.0 (61.8-99.2)

1
7
0
$121(118-124)$

$2.0(1.0-9.5)$

$12.0(1.0-20.2)$

0.573

\begin{tabular}{cc}
$\frac{\mathbf{D}}{\mathrm{E2}(2 \mathrm{~mL})(n=12)}$ & P value (overall) \\
\cline { 1 - 1 } $71.4(68.7-79.1)$ & 0.617 \\
1 & 1.000 \\
& 0.174
\end{tabular}

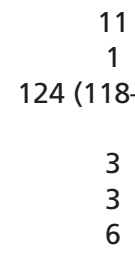

0.579

0.750

3

6

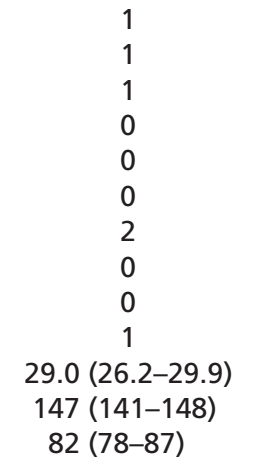

3
2
3
1
0
0
2
0
1
0

131 (129-141)

78 (74-81)

$$
\begin{gathered}
1.7(1.3-5.4) \\
1.9(1.6-5.4) \\
0.21(0.16-0.31) \\
0.10(0.10-0.40) \\
3.0(2.4-4.4) \\
11(11-11) \\
124(98.0-216) \\
72.0(60.5-73.0)
\end{gathered}
$$

$3.3(2.6-6.8)$

$4.3(3.1-13.0)$

$0.17(0.16-0.19)$

$0.10(0.10-0.20)$

$3.6(2.3-4.2)$

$11(11-11)$

115 (83.0-138)

88.5 (74.2-121)
0.501

1.000

0.401

1.000

$-$

0.378

$-$

1.000

0.485

0.766

0.131

0.590

0.609

0.494

0.063

0.208

0.996

0.892

0.312

0.449
$29.0(26.6-29.7)$

aFACT-P total score is a prostate cancer-specific quality of life score ranging from 0 to 156 , with higher scores indicating greater quality of life. 25OH-Vit D3, 25-hydroxy Vitamin D3; BMI, Body Mass Index; DBP, Diastolic blood pressure; E1, Estrone; E2, Estradiol; ECOG, Eastern Cooperative Oncology Group Performance Status; FACT-P, Functional Assessment of Cancer Therapy - Prostate; FSH, Follicle-stimulating hormone; GnRH, Gonadotrophin-releasing hormone; IQR, Interquartile range; LH, Luteinizing hormone; MI, Myocardial infaction; PCa, Prostate Cancer; SSRI, Selective serotonin reuptake inhibitor; SNRI, Selective serotonin-noradrenaline reuptake inhibitor; SBP, Systolic blood pressure; TS, Testosterone.

\section{Hot flushes}

Averaged daily hot flush scores are shown in Fig. 3A. The score declined in the E2 group, compared with the placebo group (robust MAD: -2.2 (95\% CI: -3.6 to -0.8 ; $P=0.02$ ). There was no association between ADT duration at enrolment and baseline score (data not shown). At day 28 , blinded participants were asked to indicate if their assigned treatment improved hot flushes or not. Although there was $100 \%$ compliance with hot flush diary return in completing participants, this additional question was not answered by all participants. Thirteen of 18 participants from the placebo arm answered this question, with 3 (23\%) indicating that their treatment was helpful. There was 1 drop out and 2 participants who did not answer this question in the $\mathrm{E} 2$ arm for a total of 21 participant answers of which $16(76 \%)$ indicated that the treatment was helpful $(P=0.007)$.

\section{Quality of life}

Baseline median (IQR) scores were 122 (118-133) in the E2 group and 124 (120-128) in the placebo group. At day 28, there were no significant changes between E2 and placebo groups in FACT-P total score or subscales measuring 


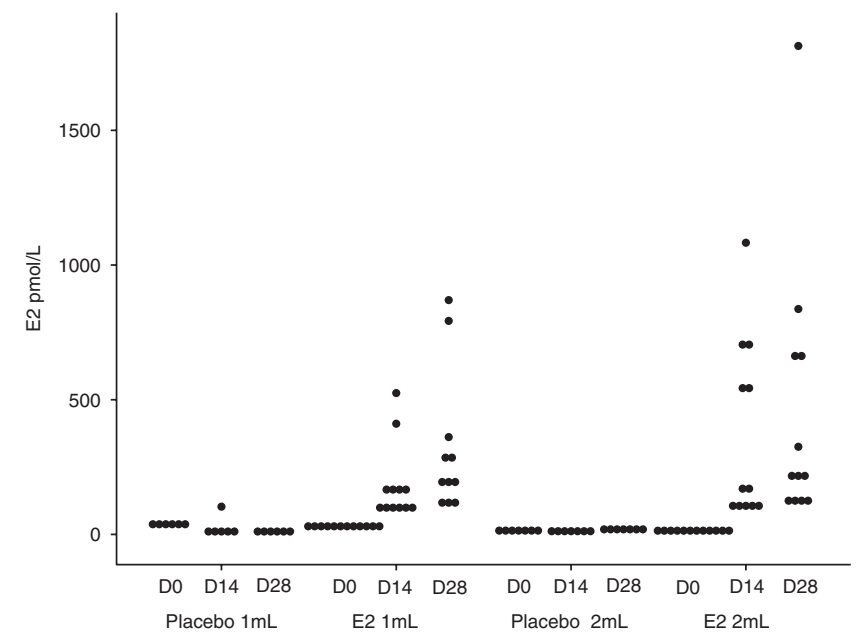

Figure 2

E2 concentration by treatment assignment and time point.

social well-being, physical well-being, emotional wellbeing, functional well-being or prostate cancer-specific symptoms (data not shown).

\section{Laboratory secondary endpoints}

For h-ematological and biochemical secondary endpoints, changes from baseline to day 28 were not different between combined E2 and placebo groups for $\mathrm{T}$ and DHT (Table 3). E2 treatment increased serum E1 and SHBG and decreased serum FSH with a possible small increase in serum LH. There was no change in haemoglobin, glucose, insulin, HOMA2-IR, IGF-1 or lipids. CTX decreased significantly in the E2 group compared to placebo, with a smaller relative increment in P1NP (Fig. 3B and C and Table 3).

\section{Adverse effects}

One participant (8\%) in the E2 $1 \mathrm{~mL}$ group, 2 (17\%) in the E2 $2 \mathrm{~mL}$ group and none in the placebo group developed grade 1 nipple tenderness by day 14 , which persisted until day 28. No participant developed breast swelling. There were no incident cardiovascular or VTE events.

\section{Discussion}

This short-term RCT found that transdermal E2 gel in daily doses of both 0.9 and $1.8 \mathrm{mg}$ increased serum E2 concentrations in men with castrate T levels due to GnRH analogue treatment. Due to the inherent indication for ADT, it was not possible to include a matched group of men with PCa not undergoing ADT. Therefore, we compared our findings to reference ranges for serum E2 that have been derived from population-based cohorts of young and older men using the same laboratory and LCMS assay.

In the Raine Birth Cohort Study of 382 healthy young Australian men aged 19-22, median serum E2 was $147 \mathrm{pmol} / \mathrm{L}$ (95\% confidence interval $57-273 \mathrm{pmol} / \mathrm{L})$ (21). In a study of 325 healthy older Australian men, median serum E2 was 86 pmol/L (95\% CI 39-153 pmol/L) (22). In a pooled analysis of over 10000 samples from 3 Australian population-based studies reporting smoothed age-specific centiles, derived male reference ranges for E2 were 38-196 pmol/L (men aged <65 years), 23-154 pmol/L (65-75 years), 22-166 pmol/L (75-85 years) and 22-174 pmol/L (>85 years) (23).

In our study, minimum and median E2 concentrations in the intervention groups were within the reported reference ranges for young and older men. Maximum

Table 2 E2 ( $\mathrm{pmol} / \mathrm{L})$ median and interquartile range by treatment assignment and time point. Values below the lower limit of detection were assigned the value of the limit of detection ( $11 \mathrm{pmol} / \mathrm{L})$.

\begin{tabular}{|c|c|c|c|c|}
\hline & A & B & $\mathbf{C}$ & D \\
\hline & Placebo $(1 \mathrm{~mL})(n=6)$ & $\mathrm{E} 2(1 \mathrm{~mL})(n=12)$ & Placebo $(2 \mathrm{~mL})(n=7)$ & $\mathrm{E} 2(2 \mathrm{~mL})(n=12)$ \\
\hline Baseline & $\begin{array}{c}11(11-11) \\
11-65\end{array}$ & $\begin{array}{c}11(11-11) \\
11-50\end{array}$ & $\begin{array}{c}11(11-11) \\
11-18\end{array}$ & $\begin{array}{c}11(11-11) \\
11-17\end{array}$ \\
\hline Day 14 & $\begin{array}{c}11(11-11) \\
11-103\end{array}$ & $\begin{array}{c}139(99-175) \\
74-525\end{array}$ & $\begin{array}{c}11(11-11) \\
11-13\end{array}$ & $\begin{array}{c}170(113-586) \\
87-1083\end{array}$ \\
\hline Day 28 & $\begin{array}{c}11(11-11) \\
11-11\end{array}$ & $\begin{array}{c}208(157-332) \\
106-870\end{array}$ & $\begin{array}{c}11(11-11) \\
11-27\end{array}$ & $\begin{array}{c}220(144-660) \\
96-1814\end{array}$ \\
\hline $\begin{array}{l}P \text { value } \\
\text { A vs B: }<0.001 \\
C \text { vs } D:<0.001\end{array}$ & & & & \\
\hline
\end{tabular}

$\mathrm{Cl}$, Confidence interval; E2, Estradiol; IQR, Interquartile range. 

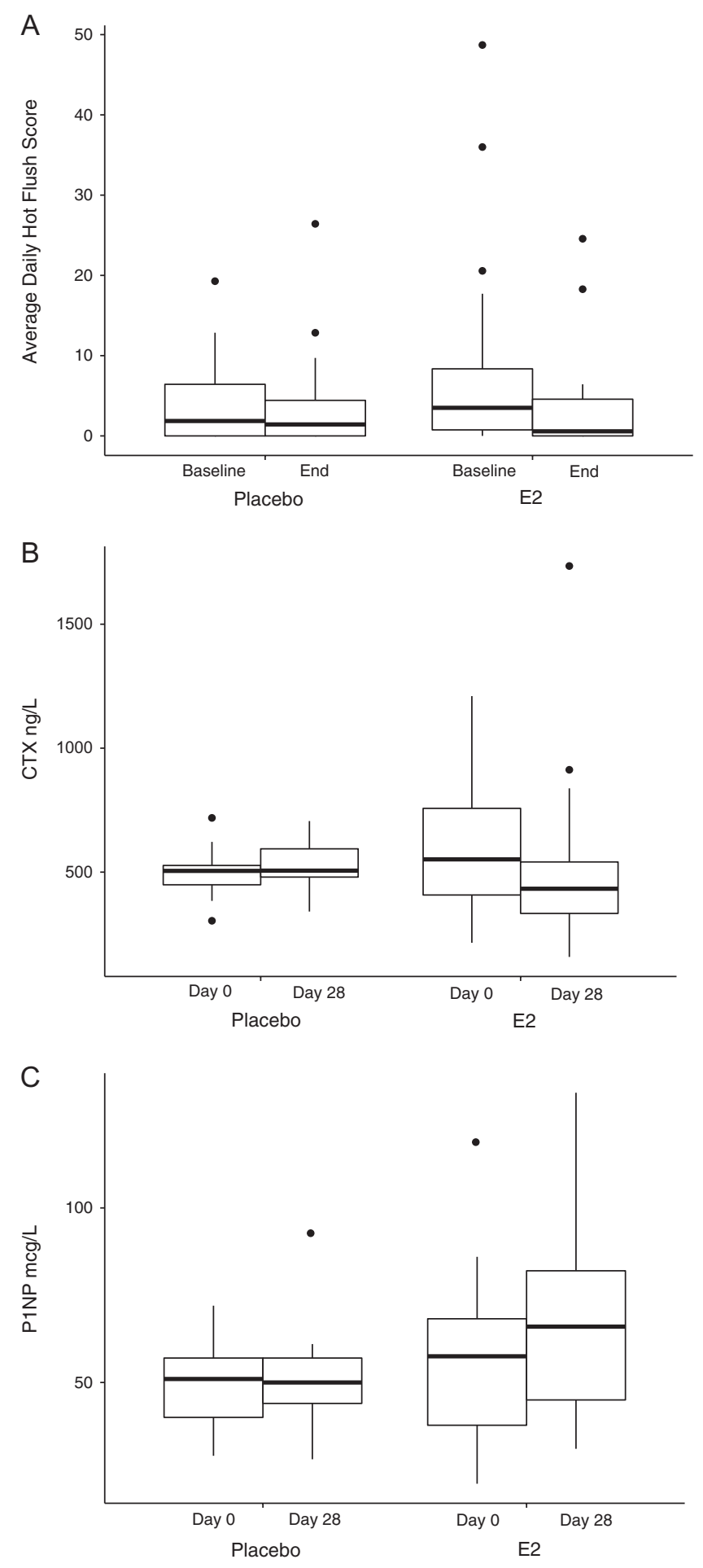

Figure 3

Box-and-whisker plots. The dark lines show the median, the lower and upper borders of the boxes are the 25th and 75th percentiles respectively. The whiskers extend 1.5 times the interquartile range below and above the bottom and top of the box. The points are individual outliers. (A) Average daily concentrations ranged from 3 to 7 times the upper reference limits. Overall, these serum E2 concentrations are substantially lower than those achieved with high-dose intramuscular polyestradiol phosphate used previously for ADT in men with $\mathrm{PCa}$, reported to range from 1400 to $4500 \mathrm{pmol} / \mathrm{L}$ (24). Reported E2 concentrations in the transdermal E2 arm of the PATCH trial were also higher (median $685 \mathrm{pmol} / \mathrm{L}$, 5th-95th percentile: 350$1788 \mathrm{pmol} / \mathrm{L})(25)$.

In the context of in vivo $\mathrm{E} 2$ synthesis by tissue aromatase occurring in healthy men, serum E2 only reflects the proportion of total synthesised E2 that has escaped local tissue metabolism and diffused into the blood (26). In the context of pharmacologic E2 add-back in men receiving $\mathrm{GnRH}$ analogues employed here, in vivo E2 synthesis is minimal due to the suppression of circulating TS, and serum E2 reflects that component of transcutaneously absorbed E2 that has not been distributed to tissues or metabolised.

We are aware of only one study assessing the effects of transdermal E2 gel on serum E2 concentrations in men. A single $2 \mathrm{mg}$ dose of transdermal E2 gel (Divigel, Orion Pharma) in 8 healthy young men produced significantly elevated serum E2 levels compared with placebo at $1 \mathrm{~h}$ and peak serum E2 levels at $2 \mathrm{~h}$ (mean $290 \mathrm{pmol} / \mathrm{L}$, range up to $514 \mathrm{pmol} / \mathrm{L})(27)$. Those findings are likely to overestimate serum E2 both because the measurements were performed with an E2 immunoassay, which overestimates male serum E2 compared with LC-MS measurements used in this study (28) as well as the fact that the non-castrate participants had higher endogenous E2 levels.

We are unable to definitively explain the mechanism for the substantial variability in circulating E2 levels observed in this study. Notably, the variability in circulating E2 levels observed here is consistent with reports of E2 gel application in postmenopausal women $(29,30)$. Even with steady-state dosing of E2 gel in women (30), and $\mathrm{T}$ gel in men (31), there are 2-fold differences between mean Cmin and Cmax and wide variability in circulating levels achieved.

hot flush score at baseline and end (median 95\% Cl). Robust mean adjusted difference between E2 group compared and placebo group was -2.2 ( -3.6 to -0.8$), P=0.02$. (B) CTX at day 0 and day 28 (median $95 \% \mathrm{Cl}$ ). Robust mean adjusted difference of E2 group compared with placebo group was $-107 \mathrm{ng} / \mathrm{L}$ $(-130$ to -84$), P<0.001$. (C) P1NP at day 0 and day 28 (median $95 \% \mathrm{Cl})$. Robust mean adjusted difference of $\mathrm{E} 2$ group compared with placebo group was $8.9 \mu \mathrm{g} / \mathrm{L}$ (5.0-12.7), $P<0.01$. 
Table 3 Change from baseline to end in men receiving E2 compared with men receiving placebo. Data are presented as median (IQR).

\begin{tabular}{|c|c|c|}
\hline & \multicolumn{2}{|c|}{ Combined E2 groups $(n=24)$} \\
\hline & D0 & $\mathrm{D} 28$ \\
\hline TS (nmol/L) & $0.16(0.13-0.20)$ & $0.17(0.13-0.24)$ \\
\hline DHT (nmol/L) & $0.17(0.17-0.17)$ & $0.17(0.17-0.17)$ \\
\hline E1 (pmol/L) & $131(91-160)$ & $706(435-923)$ \\
\hline LH (IU/L) & $0.1(0.1-0.2)$ & $0.1(0.1-0.4)$ \\
\hline FSH (IU/L) & $3.6(2.3-4.2)$ & $0.9(0.7-1.5)$ \\
\hline SHBG (nmol/L) & $65(45-70)$ & $82(51-89)$ \\
\hline FBGL (mmol/L) & $4.8(4.5-5.2)$ & $4.9(4.6-5.4)$ \\
\hline Insulin (IU/L) & $10.8(9.1-14.0)$ & $10.8(7.9-15.8)$ \\
\hline HOMA2-IR & $1.48(1.23-1.82)$ & $1.36(1.00-1.96)$ \\
\hline IGF-1 (nmol/L) & $21.3(17.6-24.1)$ & $18.8(16.2-23.7)$ \\
\hline LDL-C (mmol/L) & $2.9(2.2-3.6)$ & $2.5(2.0-3.6)$ \\
\hline $\mathrm{HDL}-\mathrm{C}(\mathrm{mmol} / \mathrm{L})$ & $1.6(1.3-1.8)$ & $1.6(1.5-1.7)$ \\
\hline TG $(\mathrm{mmol} / \mathrm{L})$ & $1.4(1.2-2.0)$ & $1.3(1.2-1.7)$ \\
\hline CTX (ng/L) & $552(408-757)$ & $433(334-541)$ \\
\hline P1NP ( $\mu g / L)$ & $58(38-68)$ & $66(45-82)$ \\
\hline
\end{tabular}

\begin{tabular}{c}
\hline Combined place \\
\hline D0 \\
\hline $0.26(0.18-0.33)$ \\
$0.17(0.17-0.17)$ \\
$144(122-198)$ \\
$0.2(0.1-0.6)$ \\
$3.0(2.1-4.5)$ \\
$70(56-86)$ \\
$5.1(4.8-5.2)$ \\
$9.9(8.9-10.9)$ \\
$1.29(1.17-1.42)$ \\
$18.2(16.2-22.9)$ \\
$2.2(1.8-3.1)$ \\
$1.6(1.4-1.9)$ \\
$1.0(0.9-1.2)$ \\
$505(449-527)$ \\
$51(40-57)$ \\
\hline
\end{tabular}

\begin{tabular}{c} 
Do groups $(n=13)$ \\
\hline $0.19(0.16-0.26)$ \\
$0.17(0.17-0.17)$ \\
$159(110-182)$ \\
$0.1(0.1-0.2)$ \\
$3.9(2.5-4.5)$ \\
$68(62-81)$ \\
$5.0(4.7-5.4)$ \\
$10.6(8.0-13.4)$ \\
$1.33(1.05-1.77)$ \\
$15.1(14.7-20.1)$ \\
$2.5(1.8-3.2)$ \\
$1.6(1.6-2.0)$ \\
$1.2(0.9-1.5)$ \\
$506(480-594)$ \\
$50(44-57)$ \\
\hline
\end{tabular}

\begin{tabular}{|c|c|}
\hline Robust MAD $(95 \% \mathrm{Cl})$ & $\begin{array}{l}P \text { value } \\
\text { For MAD }\end{array}$ \\
\hline $0.03(-0.01$ to +0.07$)$ & 0.16 \\
\hline$N / A^{a}$ & $N / A^{a}$ \\
\hline $671(583-758)$ & $<0.001$ \\
\hline $0.18(0.05-0.32)$ & 0.01 \\
\hline$-2.3(-2.9$ to -1.7$)$ & $<0.001$ \\
\hline $11.6(6.6-16.5)$ & $<0.001$ \\
\hline $0.06(-0.19$ to 0.30$)$ & 0.66 \\
\hline $1.0(-0.6$ to 0.7$)$ & 0.43 \\
\hline$-0.14(-0.32$ to 0.04$)$ & 0.14 \\
\hline$-0.37(-2.3$ to 1.5$)$ & 0.71 \\
\hline $0(-0.15$ to 0.15$)$ & 0.98 \\
\hline$-0.16(-0.26$ to -0.07$)$ & 0.001 \\
\hline$-0.19(-0.39$ to 0.01$)$ & 0.08 \\
\hline$-107(-130$ to -84$)$ & $<0.001$ \\
\hline $8.9(5.0-12.7)$ & $<0.01$ \\
\hline
\end{tabular}

aMAD was not estimable due to singularity.

$\mathrm{Cl}$, Confidence interval; CTX, beta carboxyl-terminal type 1 collagen telopeptide; D0, Day 0; D28, Day 28; DHT, Dihydrotestosterone; E1, Estrone; E2, Estradiol; FBGL, Fasting blood glucose level; FSH, Follicle-stimulating hormone; HDL-C, High-density lipoprotein cholesterol; HOMA2-IR, Updated Homeostatic Model Assessment of Insulin Resistance; IGF-1, Insulin-like growth factor 1; IQR, Interquartile range; LDL-C, Low-density lipoprotein cholesterol; LH, Luteinizing hormone; MAD, Mean adjusted difference; P1NP, pro collagen type 1 amino-terminal propeptide; SHBG, Sex hormonebinding globulin; TS, Testosterone; TG, triglycerides.

Observed variability is likely due to differences in transcutaneous absorption and metabolism of E2. In addition, while participants in our study were instructed to withhold their dose prior to their days 14 and 28 morning blood test, it is possible that some men may have applied the disallowed morning dose or had applied the gel at various times during the previous day so that the time between last dose and blood sampling may have contributed to the between-person variability in serum E2. Age did not correlate with serum E2 levels, and there was substantial overlap in levels achieved with 0.9 and $1.8 \mathrm{mg}$ doses.

Other factors contributing to E2 variability might include duration of prior hypogonadism and fat mass. In our study, BMI and duration of ADT were not correlated with E2 levels, but our study was not designed to rigorously examine these hypotheses. Future studies should do so, as they may have clinical implications regarding optimal E2 dosing considerations in such men.

Nipple tenderness was noted in 1 of 12 men receiving $0.9 \mathrm{mg}, 2$ of 12 men receiving $1.8 \mathrm{mg}$ and none using placebo gel in this study. In the PATCH trial, $75 \%$ of men receiving a high-dose transdermal E2 regimen, sufficient to induce castrate TS concentrations without GnRH analogues, experienced gynaecomastia compared to $19 \%$ of men receiving GnRH analogues (8). It is unknown to what extent long-term low-dose transdermal E2 add-back will increase the incidence of breast adverse effects above that caused by GnRH analogues alone.

The increase in circulating E1 observed here is expected given $\mathrm{E} 2$ and $\mathrm{E} 1$ undergo interconversion by 17 hydroxysteroid dehydrogenase. While E2 is the sole potent bioactive oestrogen, some observational studies have associated low E1 with adverse health outcomes. For example, in a longitudinal study of 1637 communitydwelling men, low circulating E1 but not E2 predicted a deterioration in self-rated health status (32). Larger and longer-term studies are needed to determine whether increasing circulating $\mathrm{E} 1$ has relevance for clinical outcomes in men with $\mathrm{PCa}$ receiving ADT.

\section{Hot flushes and quality of life}

There were substantial improvements in the robust MAD for daily hot flush score (Fig. 2A) with E2 treatment corroborated by the fact that more men receiving E2 reported beneficial effects than those receiving placebo. This beneficial effect occurred despite the study not selecting men for troublesome hot flushing or excluding men continuing to receive other medications for hot flushing. Yet, despite previous reports associating increased hot flushes with sleep disturbance (33) and reduced quality of life in men undergoing ADT (34, 35 ), these benefits did not translate into a detectable 
improvement in overall quality-of-life scores. This disparity may be due to a lack of power because of the short duration of this study or because baseline quality of life was already relatively high in our cohort by comparison with other men with PCa (36).

These data are consistent with an experimental study of induced hypogonadism in healthy young men with graded TS add-back with or without aromatase inhibition, indicating that E2 deficiency, not androgen deficiency, is the predominant trigger for vasomotor symptoms in men (37). However, that study inferred E2 effects indirectly by drug-induced aromatase inhibition but without a placebo control for the drug, whereas our study tested the effects of E2 in the absence of TS directly. Uncontrolled studies $(38,39)$ and a small RCT $(40)$ of diethylstilbestrol, a synthetic non-steroidal estrogen, in men undergoing non-estrogen ADT for PCa have also suggested a benefit in treating vasomotor symptoms. Furthermore, data from PATCH indicate less severe declines in global quality of life in men receiving transdermal E2 rather than GnRH analogues for treatment of locally advanced or metastatic PCa (34). This effect seemed to be driven primarily by a difference in hot flushes between arms, although men assigned to E2 had less decline in physical and sexual function at the cost of more gynecomastia.

\section{Androgens and gonadotrophins}

Although baseline TS and DHT were fully suppressed by the pre-study GnRH analogue treatment and unaffected by study E2 treatment, the low baseline serum FSH was further reduced in men receiving E2 but not placebo (Table 3). So-called 'FSH escape' has been described during long-term GnRH agonist use and attributed to loss of negative feedback on FSH from the progressive long-term decline in inhibin B seen during GnRH agonist treatment $(41,42)$. These present findings indicate that exogenous E2 has FSH-suppressive effects in men who have castrate levels of T. Whether this FSH escape is a clinically relevant phenomenon is not known. Direct roles of FSH in tumour progression and bone and metabolic side effects of ADT have been proposed (43). However, the proposed direct non-reproductive biological roles of FSH remain controversial (44).

\section{Biomarkers of bone remodelling}

There were significant reductions in CTX and increases in P1NP with E2 treatment, without changes in PTH, calcium or phosphate levels or in Vitamin D supplementation.
The acute effect with E2 add-back seen here is consistent with the fact that acutely, medical castration produces the reverse, i.e. an increase in bone resorption and transient decrease in bone formation in older otherwise healthy men (45). This pattern of change in bone remodelling suggests an antiresorptive effect of E2 add-back in men receiving GnRH analogues. In men, sex steroid deficiency results in accelerated bone remodelling, and multiple lines of evidence suggest this is largely but not solely an E2-dependent effect (46). Notably, preliminary data from the PATCH study have indicated a beneficial effect on bone density for men receiving high-dose transdermal E2 compared with GnRH analogue (25). For the alternative approach of low-dose E2 add-back in addition to GnRH analogue treatment, longer-term treatment will be necessary to determine whether this strategy will improve or preserve bone density.

\section{Hepatic protein synthesis}

Due to first pass metabolism, oral estrogens expose hepatocytes to non-physiological estrogen concentrations, while high parenteral E2 doses can achieve similar effects with supraphysiological hepatic estrogen exposure. Oral (or high parenteral) doses of estrogens increase hepatic synthesis of SHBG, clotting factors, inflammatory cytokines and binding proteins $(5,47)$. Our study showed a small, but significant increase in SHBG consistent with hepatic E2 effect. We did not measure clotting factors in this study but prior studies have observed a lack of coagulation and inflammatory factor activation with transdermal E2 (48). Moreover, high-dose transdermal E2 in the PATCH trial was not associated with an increased risk of thromboembolic events, although larger studies are needed to confirm that $\mathrm{E} 2$ treatment is safe (8).

\section{Insulin resistance and lipid metabolism}

We observed no differences in fasting lipids, blood glucose, insulin, c-peptide or insulin resistance estimated by HOMA2-IR. ADT reduces muscle mass and increases fat mass (49) and is associated with worsening insulin sensitivity (50) and incident diabetes mellitus (51). Combined deficiency of androgens and estrogens is likely to contribute to these body composition and metabolic effects (6). Theoretically, E2 add-back might have beneficial effects by reducing visceral fat accumulation $(6,52)$. While our study does not provide data to support this hypothesis, it may have been too short to detect such effects. 


\section{Strengths and limitations of the study}

Strengths of the study include its unique randomised design of relatively low-dose transdermal E2 add-back given to men with prostate cancer receiving GnRH analogue-based ADT, which is in contrast to using highdose E2 as a sole mode of ADT (8). The latter strategy leads to supraphysiologic E2 concentrations, which may be associated with an increased risk of undesirable effects. Moreover, the study design allowed us to evaluate direct actions of $\mathrm{E} 2$ in the absence of $\mathrm{T}$, whereas previous seminal work inferred E2 effects indirectly by druginduced aromatase inhibition $(6,37)$. In addition, a relatively large number of outcomes were assessed, and E2 concentrations were measured by validated LC-MS/ MS technology.

The principal limitations are the short duration and small sample size of this study, preventing definitive conclusions about the presence or durability of any clinical benefits from a strategy of E2 add-back. Our study was too short to evaluate the oncologic outcomes of E2 treatment. While preclinical data are not conclusive (9), a systematic review of 20 RCTs of the use of high-dose parenteral E2 as ADT for locally advanced or metastatic PCa found no evidence that E2 treatment differed in efficacy from GnRH analogues or orchiectomy when comparing overall mortality (53).

\section{Conclusion}

Our study demonstrates that in men who have castrate levels of both E2 and TS, daily transdermal E2 0.9-1.8 mg increases median serum E2 concentrations into the reference range reported for healthy young or older men, but with substantial variability. E2 gel treatment reduces vasomotor symptoms and bone remodelling in older men with $\mathrm{PCa}$, and further suppresses partially suppressed serum FSH in the absence of TS. These findings provide a rationale to test the hypothesis that such E2 add-back will benefit men by mitigating bone and, possibly metabolic adverse effects of ADT due to GnRH analogue treatment in a longer-term RCT. Further trials would reasonably proceed with a $0.9 \mathrm{mg}$ E2 dose rather than $1.8 \mathrm{mg}$ dose, based on the largely overlapping serum levels achieved here and the hypothesis that a lower dose may result in fewer adverse effects.

\section{Declaration of interest}

Mathis Grossmann has received research funding from Bayer Pharma, Novartis, Weight Watchers, Lilly and speaker's honoraria from Besins Healthcare. Ada Cheung has received speaker's honoraria from Merck,
Sharpe, Dohme and Astra Zeneca. David Handelsman has received institutional funding for investigator-initiated testosterone pharmacology research from Besins Healthcare and Lawley and has provided expert testimony in anti-doping tribunals and testosterone litigation. Nicholas Russell, Rudolf Hoermann, and Jeffrey D Zajac declare that they have no conflict of interest.

\section{Funding}

This trial was funded by a project grant from the National Health and Medical Research Council (\#APP1099173).

Author contribution statement

N R contributed to study design, coordinated the trial, analysed the results and wrote the manuscript; $\mathrm{R} \mathrm{H}$ performed statistical analysis and revised the manuscript; $M G$ designed the trial, analysed the results and revised the manuscript; D J H performed the LC-MS assays and revised the manuscript; $\mathrm{M} C$ performed the randomisation and revised the manuscript; A S C assisted in recruitment and revised the manuscript; J D Z contributed to study design.

\section{Acknowledgements}

NR is supported by an Australian Postgraduate Award. AS is supported by an Endocrine Society of Australia Postdoctoral Award.

\section{References}

1 Mottet N, Bellmunt J, Bolla M, Briers E, Cumberbatch MG, De Santis M, Fossati N, Gross T, Henry AM, Joniau S et al. EAU-ESTROSIOG guidelines on prostate cancer. Part 1: screening, diagnosis, and local treatment with curative intent. European Urology $2017 \mathbf{7 1}$ 618-629. (https://doi.org/10.1016/j.eururo.2016.08.003)

2 Cornford P, Bellmunt J, Bolla M, Briers E, De Santis M, Gross T, Henry AM, Joniau S, Lam TB, Mason MD et al. EAU-ESTRO-SIOG guidelines on prostate cancer. Part II: treatment of relapsing, metastatic, and castration-resistant prostate cancer. European Urology 201771 630-642. (https://doi.org/10.1016/j.eururo.2016.08.002)

3 Byar DP. The Veterans Administration Cooperative Urological Research Group's studies of cancer of the prostate. Cancer 197332 1126-1130. (https://doi.org/10.1002/10970142(197311)32:5<1126::AID-CNCR2820320518>3.0.CO;2-C)

4 Leuprolide Study Group. Leuprolide versus diethylstilbestrol for metastatic prostate cancer. New England Journal of Medicine 1984311 1281-1286. (https://doi.org/10.1056/NEJM198411153112004)

5 Schoultz von B, Carlström K, Collste L, Eriksson A, Henriksson P, Pousette A \& Stege R. Estrogen therapy and liver function metabolic effects of oral and parenteral administration. Prostate 1989 14 389-395. (https://doi.org/10.1002/pros.2990140410)

6 Finkelstein JS, Lee H, Burnett-Bowie SAM, Pallais JC, Yu EW, Borges LF, Jones BF, Barry CV, Wulczyn KE, Thomas BJ et al. Gonadal steroids and body composition, strength, and sexual function in men. New England Journal of Medicine 2013369 1011-1022. (https:// doi.org/10.1056/NEJMoa1206168)

7 Ockrim J, Lalani EN \& Abel P. Therapy insight: parenteral estrogen treatment for prostate cancer - a new dawn for an old therapy. Nature Clinical Practice and Oncology 20063 552-563. (https://doi. org/10.1038/ncponc0602)

8 Langley RE, Cafferty FH, Alhasso AA, Rosen SD, Sundaram SK, Freeman SC, Pollock P, Jinks RC, Godsland IF, Kockelbergh R et al. Cardiovascular outcomes in patients with locally advanced and metastatic prostate cancer treated with luteinising- hormonereleasing-hormone agonists or transdermal oestrogen: the 
randomised, phase 2 MRC PATCH trial. Lancet Oncology 201314 306-316. (https://doi.org/10.1016/S1470-2045(13)70025-1)

9 Russell N, Cheung A \& Grossmann M. Estradiol for the mitigation of adverse effects of androgen deprivation therapy. Endocrine-Related Cancer 201724 R297-R313. (https://doi.org/10.1530/ERC-17-0153)

10 Komesaroff PA, Fullerton M, Esler MD, Dart A, Jennings G \& Sudhir K. Low-dose estrogen supplementation improves vascular function in hypogonadal men. Hypertension 200138 1011-1016. (https://doi.org/10.1161/hy1101.095006)

11 Komesaroff PA, Fullerton M, Esler MD, Jennings G \& Sudhir K. Oestrogen supplementation attenuates responses to psychological stress in elderly men rendered hypogonadal after treatment for prostate cancer. Clinical Endocrinology 200256 745-753. (https://doi. org/10.1046/j.1365-2265.2002.01542.x)

12 Matousek RH \& Sherwin BB. A randomized controlled trial of addback estrogen or placebo on cognition in men with prostate cancer receiving an antiandrogen and a gonadotropin-releasing hormone analog. Psychoneuroendocrinology 201035 215-225. (https://doi. org/10.1016/j.psyneuen.2009.06.012)

13 Loprinzi CL, Michalak JC, Quella SK, O'Fallon JR, Hatfield AK, Nelimark RA, Dose AM, Fischer T, Johnson C \& Klatt NE. Megestrol acetate for the prevention of hot flashes. New England Journal of Medicine 1994331 347-352. (https://doi.org/10.1056/ NEJM199408113310602)

14 NIH National Cancer Institute. Common terminology criteria for adverse events version 4.03. 2010.

15 Harwood DT \& Handelsman DJ. Development and validation of a sensitive liquid chromatography-tandem mass spectrometry assay to simultaneously measure androgens and estrogens in serum without derivatization. Clinica Chimica Acta 2009409 78-84. (https://doi. org/10.1016/j.cca.2009.09.003)

16 Wallace TM, Levy JC \& Matthews DR. Use and abuse of HOMA modeling. Diabetes Care 200427 1487-1495. (https://doi org/10.2337/diacare.27.6.1487)

17 Simon JA, Bouchard C, Waldbaum A, Utian W, Zborowski J \& Snabes MC. Low dose of transdermal estradiol gel for treatment of symptomatic postmenopausal women: a randomized controlled trial. Obstetrics and Gynecology 2007109 588-596. (https://doi. org/10.1097/01.AOG.0000254160.62588.41)

18 R Core Team. R: A Language and Environment for Statistical Computing. Vienna, Austria: R-Foundation for Statistical Computing.

19 Koller M. Robustlmm: an R package for robust estimation of linear mixed-effects models. Journal of Statistical Software 201675 1-24. (https://doi.org/10.18637/jss.v075.i06)

20 Kuznetsova A, Brockhoff PB \& Christensen RHB. ImerTestPackage: tests in linear mixed effects models. Journal of Statistical Software 2017 82 1-26. (https://doi.org/10.18637/jss.v082.i13)

21 Hart RJ, Doherty DA, McLachlan RI, Walls ML, Keelan JA, Dickinson JE, Skakkebaek NE, Norman RJ \& Handelsman DJ. Testicular function in a birth cohort of young men. Human Reproduction 201530 2713-2724. (https://doi.org/10.1093/humrep/ dev244)

22 Sartorius G, Spasevska S, Idan A, Turner L, Forbes E, Zamojska A, Allan CA, Ly LP, Conway AJ, McLachlan RI et al. Serum testosterone, dihydrotestosterone and estradiol concentrations in older men self-reporting very good health: the healthy man study. Clinical Endocrinology 201277 755-763. (https://doi.org/10.1111/j.13652265.2012.04432.x)

23 Handelsman DJ, Yeap B, Flicker L, Martin S, Wittert GA \& Ly LP. Age-specific population centiles for androgen status in men. European Journal of Endocrinology 2015173 809-817. (https://doi.org/10.1530/ EJE-15-0380)

24 Henriksson P, Carlström K, Pousette A, Gunnarsson PO, Johansson CJ, Eriksson B, Altersgård-Brorsson AK, Nordle O \& Stege R. Time for revival of estrogens in the treatment of advanced prostatic carcinoma? Pharmacokinetics, and endocrine and clinical effects, of a parenteral estrogen regimen. Prostate 199940 76-82. (https://doi.org/10.1002/(SICI)1097-0045(19990701)40:2<76::AIDPROS2>3.0.CO;2-Q)

25 Langley RE, Kynaston HG, Alhasso AA, Duong T, Paez EM, Jovic G, Scrase CD, Robertson A, Cafferty F, Welland A et al. A randomised comparison evaluating changes in bone mineral density in advanced prostate cancer: luteinising hormone-releasing hormone agonists versus transdermal oestradiol. European Urology 201669 1016-1025. (https://doi.org/10.1016/j.eururo.2015.11.030)

26 Simpson ER. Sources of estrogen and their importance. Journal of Steroid Biochemistry and Molecular Biology 200386 225-230. (https:// doi.org/10.1016/S0960-0760(03)00360-1)

27 Eisenegger C, Eckardstein von A, Fehr E \& Eckardstein von S. Pharmacokinetics of testosterone and estradiol gel preparations in healthy young men. Psychoneuroendocrinology 201338 171-178. (https://doi.org/10.1016/j.psyneuen.2012.05.018)

28 Handelsman DJ, Newman JD, Jimenez M, McLachlan R, Sartorius G $\&$ Jones GRD. Performance of direct estradiol immunoassays with human male serum samples. Clinical Chemistry 201460 510-517. (https://doi.org/10.1373/clinchem.2013.213363)

29 Aspen Pharmacare Australia Pty Limited. Product Information Sandrena 0.1\% Gel, pp 1-10, 2013.

30 Azur Pharma International II Ltd. Elestrin Product Information, pp 1-33. Philadelphia, PA: Azur Pharma, 2012

31 Swerdloff RS, Pak Y, Wang C, Liu PY, Bhasin S, Gill TM, Matsumoto AM, Pahor M, Surampudi P \& Snyder PJ. Serum testosterone (T) level variability in T gel-treated older hypogonadal men: treatment monitoring implications. Journal of Clinical Endocrinology and Metabolism 2015100 3280-3287. (https://doi. org/10.1210/JC.2015-1542)

32 Hsu B, Cumming RG, Blyth FM, Naganathan V, Le Couteur DG, Seibel MJ, Waite LM \& Handelsman DJ. Longitudinal and crosssectional relationships of circulating reproductive hormone levels to self-rated health and health-related quality of life in community-dwelling older men. Journal of Clinical Endocrinology and Metabolism 201499 1638-1647. (https://doi.org/10.1210/ jc.2013-3984)

33 Gonzalez BD, Small BJ, Cases MG, Williams NL, Fishman MN, Jacobsen PB \& Jim HSL. Sleep disturbance in men receiving androgen deprivation therapy for prostate cancer: the role of hot flashes and nocturia. Cancer 2018124 499-506. (https://doi.org/10.1002/ cncr.31024)

34 Gilbert DC, Duong T, Kynaston HG, Alhasso AA, Cafferty FH, Rosen SD, Kanaga-Sundaram S, Dixit S, Laniado M, Madaan S et al. Quality-of-life outcomes from the prostate adenocarcinoma: transcutaneous hormones (PATCH) trial evaluating luteinising hormone-releasing hormone agonists versus transdermal oestradiol for androgen suppression in advanced prostate cancer BJU International 2017119 667-675. (https://doi.org/10.1111/ bju.13687)

35 Cheung AS, de Rooy C, Hoermann R, Lim Joon D, Zajac JD \& Grossmann M. Quality of life decrements in men with prostate cancer undergoing androgen deprivation therapy. Clinical Endocrinology 201786 388-394. (https://doi.org/10.1111/ cen.13249)

36 Esper P, Mo F, Chodak G, Sinner M, Cella D \& Pienta KJ. Measuring quality of life in men with prostate cancer using the Functional Assessment of Cancer Therapy-prostate instrument. Urology $1997 \mathbf{5 0}$ 920-928. (https://doi.org/10.1016/s0090-4295(97)00459-7)

37 Taylor AP, Lee H, Webb ML, Joffe H \& Finkelstein JS. Effects of testosterone and estradiol deficiency on vasomotor symptoms in hypogonadal men. Journal of Clinical Endocrinology and Metabolism 2016101 3479-3486. (https://doi.org/10.1210/jc.2016-1612)

38 Miller JI \& Ahmann FR. Treatment of castration-induced menopausal symptoms with low dose diethylstilbestrol in men with advanced prostate cancer. Urology $1992 \mathbf{4 0} 499-502$. 
39 Smith JA. A prospective comparison of treatments for symptomatic hot flushes following endocrine therapy for carcinoma of the prostate. Journal of Urology 1994152 132-134. (https://doi. org/10.1016/S0022-5347(17)32835-5)

40 Atala A, Amin M \& Harty JI. Diethylstilbestrol in treatment of postorchiectomy vasomotor symptoms and its relationship with serum follicle-stimulating hormone, luteinizing hormone, and testosterone. Urology 199239 108-110. (https://doi. org/10.1016/0090-4295(92)90264-W)

41 Bhasin S, Berman N \& Swerdloff RS. Follicle-stimulating hormone (FSH) escape during chronic gonadotropin-releasing hormone $(\mathrm{GnRH})$ agonist and testosterone treatment. Journal of Andrology 199415 386-391. (https://doi.org/10.1002/j.1939-4640.1994. tb00470.x)

42 Eldar-Geva T, Liberty G, Chertin B, Fridmans A, Farkas A, Margalioth EJ \& Spitz IM. Relationships between FSH, inhibin $\mathrm{B}$, anti-Mullerian hormone, and testosterone during long-term treatment with the GnRH-agonist histrelin in patients with prostate cancer. European Journal of Endocrinology 2010162 177-181. (https:// doi.org/10.1530/EJE-09-0366)

43 Liu P, Ji Y, Yuen T, Rendina-Ruedy E, DeMambro VE, Dhawan S, AbuAmer W, Izadmehr S, Zhou B, Shin AC et al. Blocking FSH induces thermogenic adipose tissue and reduces body fat. Nature $2017 \mathbf{5 4 6}$ 107-112. (https://doi.org/10.1038/nature22342)

44 Allan CM, Kalak R, Dunstan CR, McTavish KJ, Zhou H, Handelsman DJ \& Seibel MJ. Follicle-stimulating hormone increases bone mass in female mice. PNAS 2010107 22629-22634. (https:// doi.org/10.1073/pnas.1012141108)

45 Falahati-Nini A, Riggs BL, Atkinson EJ, O'Fallon WM, Eastell R \& Khosla S. Relative contributions of testosterone and estrogen in regulating bone resorption and formation in normal elderly men. Journal of Clinical Investigation 2000106 1553-1560. (https://doi. org/10.1172/JCI10942)

46 Finkelstein JS, Lee H, Leder BZ, Burnett-Bowie SAM, Goldstein DW, Hahn CW, Hirsch SC, Linker A, Perros N, Servais AB et al. Gonadal steroid-dependent effects on bone turnover and bone mineral density in men. Journal of Clinical Investigation 2016126 1114-1125. (https://doi.org/10.1172/JCI84137)

47 Chetkowski RJ, Meldrum DR, Steingold KA, Randle D, Lu JK, Eggena P, Hershman JM, Alkjaersig NK, Fletcher AP \& Judd HL. Biologic effects of transdermal estradiol. New England Journal of Medicine 1986314 1615-1620. (https://doi.org/10.1056/ NEJM198606193142505)

48 Ockrim JL, Lalani EN, Kakkar AK \& Abel PD. Transdermal estradiol therapy for prostate cancer reduces thrombophilic activation and protects against thromboembolism. Journal of Urology $2005 \mathbf{1 7 4}$ 527-533.

49 Greenspan SL, Coates P, Sereika SM, Nelson JB, Trump DL \& Resnick NM. Bone loss after initiation of androgen deprivation therapy in patients with prostate cancer. Journal of Clinical Endocrinology and Metabolism 200590 6410-6417. (https://doi. org/10.1210/jc.2005-0183)

50 Cheung AS, Hoermann R, Dupuis P, Joon DL, Zajac JD \& Grossmann M. Relationships between insulin resistance and frailty with body composition and testosterone in men undergoing androgen deprivation therapy for prostate cancer. European Journal of Endocrinology 2016175 229-237. (https://doi.org/10.1530/EJE16-0200)

51 Keating NL, O’Malley AJ \& Smith MR. Diabetes and cardiovascular disease during androgen deprivation therapy for prostate cancer. Journal of Clinical Oncology 200624 4448-4456. (https://doi. org/10.1200/JCO.2006.06.2497)

52 Jeffery E, Wing A, Holtrup B, Sebo Z, Kaplan JL, Saavedra-Peña R, Church CD, Colman L, Berry R \& Rodeheffer MS. The adipose tissue microenvironment regulates depot-specific adipogenesis in obesity. Cell Metabolism 201624 142-150. (https://doi. org/10.1016/j.cmet.2016.05.012)

53 Norman G, Dean ME, Langley RE, Hodges ZC, Ritchie G, Parmar MKB, Sydes MR, Abel P \& Eastwood AJ. Parenteral oestrogen in the treatment of prostate cancer: a systematic review. British Journal of Cancer 200898 697-707. (https://doi.org/10.1038/ sj.bjc.6604230)

Received 26 December 2017

Revised version received 7 March 2018

Accepted 15 March 2018 